\title{
T4 early promoter strength probed in vivo with unribosylated and ADP-ribosylated Escherichia coli RNA polymerase: a mutation analysis
}

\author{
Nicole Sommer, ${ }^{1}$ Vida Salniene, ${ }^{2}$ Egle Gineikiene, ${ }^{2}$ Rimas Nivinskas ${ }^{2}$ \\ and Wolfgang Rüger ${ }^{1}$
}

\footnotetext{
1 Arbeitsgruppe Molekulare Genetik, Fakultät für Biologie, Ruhr-Universität Bochum, D-44780 Bochum, Germany

2 Institute of Biochemistry, Laboratory of Gene Engineering, Vilnius 2600, Lithuania
}

Author for correspondence: Wolfgang Rüger. Tel: +49 23432 23102. Fax: +49 2343214195. e-mail: wolfgang.rueger@ruhr-uni-bochum.de

The consensus sequence of T4 early promoters differs in length, sequence and degree of conservation from that of Escherichia coli $\sigma^{70}$ promoters. The enzyme interacting with these promoters, and transcribing the T4 genome, is native host RNA polymerase, which is increasingly modified by the phage-encoded ADP-ribosyltransferase, Alt. T4 early transcription is a very active process, possibly out-competing host transcription. The much stronger T4 promoters enhance viral transcription by a factor of at least two and the Alt-catalysed ADP-ribosylation of the host enzyme triggers an additional enhancement, again by a factor of about two. To address the question of which promoter elements contribute to the increasing transcriptional activity directed towards phage genes, the very strong E. coli promoter, Ptac, was sequentially mutated towards the sequence of the T4 early promoter consensus. Second, mutations were introduced into the highly conserved regions of the T4 early promoter, P8.1. The co-occurrence of the promoter-encoding plasmid pKWIII and vector PTKRI, which expresses Alt in E. coli, constitutes a test system that allows comparison of the transcriptional activities of phage and bacterial promoters, in the presence of native, or alternatively ADP-ribosylated RNA polymerase. Results reveal that T4 early promoters exhibit a bipartite structure, capable of strong interaction with both types of RNA polymerase. The $-10,-16,-42$ and $\mathbf{- 5 2}$ regions are important for transcript initiation with the native polymerase. To facilitate acceleration of transcription, the ADP-ribosylated enzyme requires not only the integrity of the $-10,-16$ and -35 regions, but also that of position -33 , and even more importantly, maintenance of the upstream promoter element at position $\mathbf{- 4 2}$. The latter positions introduced into the $E$. coli Ptac promoter render this mutant promoter responsive to Alt-ADPribosylated RNA polymerase, like T4 early promoters.

Keywords: ADP-ribosylation, modified RNA polymerase, T4 phage, prokaryotic promoter, mutation analysis

\section{INTRODUCTION}

For many years, elucidation of the regulatory steps driving the temporally controlled transcription of $\mathrm{T} 4$ phage genes has provided a challenge for research. Once T4 DNA has entered the host cell, about 40 T4 early promoters must compete with more than 650 host promoters for the $\sigma^{70}$ RNA polymerase in the cellular pool (Blattner et al., 1997; Perez-Rueda et al., 1998;

Abbreviations: Alt, T4 ADP-ribosyltransferase; PU, promoter unit.
Salgado et al., 1999). The virulence of T4 phage is proof that this task is accomplished with high efficiency. Though infection by the phage leads to rapid inhibition of transcription of the host genes, T4-specific transcription nevertheless depends on host RNA polymerase. The enzyme is sequentially modified by phageencoded polypeptides and with the appearance of viral regulatory factors, host RNA polymerase undergoes structural rearrangements, leading to a time-dependent activation of three classes of $\mathrm{T} 4$ promoters: early, middle and late. 


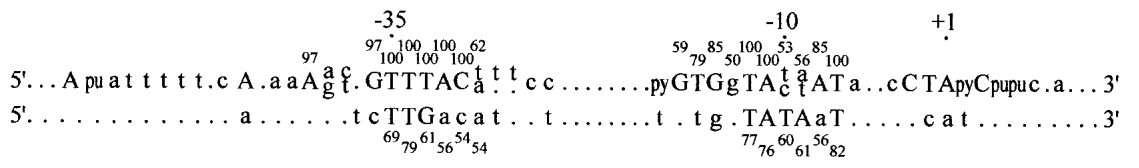

Fig. 1. Consensus sequence of T4 early promoters in the upper line is compared to that of $E$. coli in the lower line. Upper case letters represent highly conserved bases while lower case letters correspond to less conserved positions. Two bases at the same position indicate one of two alternatives (pu, purines; py, pyrimidines). The percentage of the most frequent base at each position is given above and below the corresponding consensus sequence (Wilkens \& Rüger, 1994).
It has been assumed that $\mathrm{T} 4$ early promoters are similar to bacterial promoters. However, cloning these promoters, using a vector specifically constructed to select and sequence these elements (Liebig \& Rüger, 1989; Wilkens \& Rüger, 1994), has revealed a consensus sequence that differs in sequence and length from that of the Escherichia coli promoters (Fig. 1). The most prominent deviations are a -10 region extended to more than 10 bp by a tGTGG motif [a feature which appears to correspond to the -16 region described for E. coli promoters by Siebenlist et al. (1980) and Voskuil \& Chambliss (1998)], a characteristic and absolutely conserved -35 region and significant conservation of two adenine tracts (up to $\mathrm{A}_{6}$ ) centred at position -42 , as well as at position -52 , which are separated by thymine runs of similar length (Liebig \& Rüger, 1989). Most of the T4 early promoters carry internal palindromic sequences and show a strikingly high information content attributable to their length and conservation (Schneider et al., 1986). This feature may meet the requirements of a virus infecting a number of different host bacteria in its natural environment and consequently interacting with RNA polymerases of possibly non-identical amino acid sequences, and also with RNA polymerases carrying phage-induced modifications.

The first modification made to the E. coli RNA polymerase after T4 infection is the ADP-ribosylation of $\operatorname{Arg}^{265}$, at one of the two $\alpha$-carboxy-terminal domains, by the phage gene product, Alt (Horvitz, 1974; Goff, 1974; Rohrer et al., 1975; Ovchinnikov et al., 1977). About 40 copies of this protein, which also acts as an internal protein of the phage head, enter the host cell together with the infecting phage DNA (Black et al., 1994). We show that T4 early promoters are bipartite elements, interacting at least twice as efficiently with native RNA polymerase as do the strongest E. coli promoters. The sequence characteristics that facilitate these attributes of the $\mathrm{T} 4$ promoters are the conserved and extended -10 regions, the absolutely conserved -35 regions and, to a minor extent, the A runs centred around positions -42 and -52 (promoter upstream elements). T4 promoter strength is further increased by a factor of about two, after ADP-ribosylation of the enzyme, as catalysed by the T4 gene product, Alt. This differential performance again depends on the integrity of the extended -10 region. However, more importantly, the modified RNA polymerase and T4 early promoter regions interact at the T4-specific -35 region, (position -33 seems to be of special importance) and at the -42 region. Transcription from the E. coli promoter Ptac, after introduction of these sequence elements by mutation, is enhanced if probed with ADP-ribosylated RNA polymerase.

\section{METHODS}

Bacterial strains, phages and plasmids. A brief description of the bacterial strains, phages and plasmids used in this study is given in Table 1.

Construction of M13HDL19/Ptac. Plasmid pBTac1 (Boehringer Mannheim) contains the tac promoter sequence (de Boer et al., 1983). This artificial, chimeric promoter matches the consensus sequence established for E. coli promoters. It carries the -10 region of the lacUV5 promoter and the -35 region of the trp promoter. This promoter fragment was excised from pBTac1 with endonucleases HaeIII and BamHI and cloned into the HincII and BamHI sites of M13HDL17 (Liebig \& Rüger, 1989), resulting in phage vector M13HDL17/Ptac.

Recombinant bacteriophages M13HDL17mp18/Ptac and M13HDL17mp18/P8.1 and the construction of Ptac and P8.1 promoter mutants. Bacteriophage vector M13HDL17 (Liebig \& Rüger, 1989), suitable for cloning and sequencing strong promoters, was constructed as a derivative of phage M13mp8 which carries amber mutations in two genes (Norrander et al., 1983; Yanisch-Perron et al., 1985). These amber mutations prevent DNA single-strand formation and phage M13 maturation on E. coli su strains. To restore the respective wild-type alleles, Cfr10I-BglI DNA fragments carrying the promoter-probe cassettes for promoters Ptac and P8.1, respectively, were cleaved out from phage vectors M13HDL17/ Ptac and M13HDL17/P8.1 and were recloned into the Cfr10I and $B g l$ I sites of M13mp18. This step yielded phage vectors M13HDL17mp18/Ptac and M13HDL17mp18/P8.1, and upon transformation gave single-stranded progeny phage.

The Ptac and P8.1 promoter mutants were generated by sitedirected mutagenesis according to Kunkel (Kunkel, 1985; Kunkel et al., 1987). Uracil-containing single-stranded DNAs, isolated from bacteriophages M13HDL17mp18/Ptac and M13HDL17mp18/P8.1 grown in and isolated from E. coli CJ236 (or BW313) provided the starting material for mutagenesis. Different synthetic oligonucleotides, carrying 'mutated' bases as listed in Table 2 were hybridized to their corresponding template DNAs and extended in an in vitro reaction. The resulting double-stranded DNAs, containing the mismatch sequences designed to change defined bases within the Ptac and P8.1 promoter sequences, were transformed into E. coli CMK603. The resulting progeny phage carrying the 
Table 1. Bacterial strains, phages and plasmids used in this study

\begin{tabular}{|c|c|c|}
\hline Strain/phage/plasmid & Description & Source/reference \\
\hline \multicolumn{3}{|l|}{ Bacterial strains: } \\
\hline E. coli CMK603 & $\begin{array}{l}\text { thi-1 thr-1 leuB6 lacY1 tonA21 supE44 hsdR } R_{\mathrm{k}} \text { hsd } \mathrm{M}_{\mathrm{k}} \\
\text { slac-pro } \mathrm{F}^{\prime}\left[\text { traD6 proAB }{ }^{+} \text {lacZ } \Delta \mathrm{M} 15\right]\end{array}$ & K. Rand, Cambridge \\
\hline E. coli CJ236 & dut -1 ung-1 thi-1 relA1/pCJ105 $\left(\right.$ cam $\left.^{\mathrm{R}} \mathrm{F}^{\prime}\right)$ & Kunkel et al. (1987) \\
\hline E. coli BW313 & HfrKL16 PO/45 [lysA(61-62)] dut-1 ung-1 thi-1 relA1 & Kunkel et al. (1987) \\
\hline \multicolumn{3}{|l|}{ Bacteriophages: } \\
\hline M13HDL17 & $\begin{array}{l}\text { Phage vector for cloning and sequencing of strong } \\
\text { promoters }\end{array}$ & Liebig \& Rüger (1989) \\
\hline M13mp18 & M13 cloning vector & $\begin{array}{l}\text { Yanisch-Perron et al. (1983); } \\
\text { Pharmacia Biotech }\end{array}$ \\
\hline M13HDL17/Ptac & Phage vector containing Ptac promoter & This study \\
\hline M13HDL17/P8.1 & Phage vector containing P8.1 promoter & Liebig \& Rüger (1989) \\
\hline M13HDL17mp18/Ptac & Phage vector for Ptac promoter mutagenesis & This study \\
\hline M13HDL17mp18/P8.1 & Phage vector for P8.1 promoter mutagenesis & This study \\
\hline \multicolumn{3}{|l|}{ Plasmids: } \\
\hline pBTac1 & tac-promoter-carrying plasmid & Boehringer Mannheim \\
\hline pKWIII & Promoter cloning and test plasmid & Wilkens \& Rüger (1994) \\
\hline pTKRI & Alt-expressing plasmid & Koch et al. (1995) \\
\hline
\end{tabular}

Table 2. Mutagenesis primers used in this study

\begin{tabular}{|clcl|}
\hline Primer & \multicolumn{1}{c|}{ Sequence $\left(\mathbf{5}^{\prime}-\mathbf{3}^{\prime}\right)$} & Primer & \multicolumn{1}{c|}{ Sequence $\left(\mathbf{5}^{\prime}-\mathbf{3}^{\prime}\right)$} \\
\hline 1 & CGATGATTAATTGTAAACAGCTC & 12 & CCGATGATT(A/T)AATTGTAAACAGC \\
2 & CACACATTATACCAGCCGATG & 13 & TACCACCCGA(A/T)TGATTAATTGTA \\
3 & CAATTCCACACATTGTACGAGC & 14 & CATTATACC(G/C)CGGATGATTAAT \\
4 & CACATTATACGACCCGATG & 15 & CCGAGGAAGGAGACAACTTTTTATCG \\
5 & CACACATTGTACGACCCGATG & 16 & CGATAAAAAGTTGTCTCCTTCCTCGG \\
6 & CACACATT(G/A)TACCA(C/G)CCGATG & 17 & GGAAGTAAACAACTGTGTATCG \\
7 & CACACATTATACCACCCGATG & 18 & TATCGTGGTGTTGGAAGAG \\
8 & CACACATAGTACCACCCGATG & 21 & CAACAGCTTTTTTCAGAATATTTGC \\
9 & CACATTGTACCACCCGATGATTA & 22 & CCGAGGAAGTCAACAACTTTTTATCG \\
10 & AAACAGCTTTTTTCAGAATATTTGC & 23 & CCTCAGTAGCTATCGTCGTATAGTACC \\
11 & CAGCTTTTGTGAGAATATTTGCC & 24 & CCTCAGTAGCTATCCCCGGATAGTACC \\
\hline
\end{tabular}

promoter mutation were isolated according to Sambrook et al. (1989). Their DNA was sequenced to verify the mutation introduced (Sanger et al., 1977). Mutated promoters were PCR-amplified (Saiki et al., 1988) using synthetic primers HDL17-rev (5'-CATCAAACAGGATTTTCG-3') and HDL17/pKW-rev (5'-TGGAATGTGTAAGAGCG-3'). DNA fragments amplified from bacteriophage M13HDL17mp18/ Ptac, as well as its mutant derivatives, were digested with StuI and BamHI. In the case of promoter P8.1, the PCRamplified DNA fragments were digested with EcoRV and BamHI. The resulting DNA fragments carrying the mutated Ptac or P8.1 promoters were inserted into SmaI and BamHI sites of vector pKWIII. All mutagenesis primers listed in Table 2 were synthesized and purchased from Life Technologies.

The promoter mutants M123-P, M123-PC, M1234-P, M1234PC, M124-P and M1-PC were obtained by an improved PCRmediated site-directed mutagenesis according to Barettino et al. (1994). In the first PCR reaction we employed the mutagenic primers and the promoter-flanking primer HDL17-rev to generate a DNA fragment (megaprimer) from M13HDL-
$17 \mathrm{mp} 18 /$ Ptac. With the resulting megaprimer and the two promoter-flanking primers, HDL17-rev and HDL17/ pKW-rev, a second PCR reaction was executed, in which pKWIII/Ptac served as template.

Promoter mutant P8.1-35a was obtained according to Aiyar \& Leis (1993). Using primer pair 15 (see above) and T7 (5'AATACGACTCACTATAGG-3'), as well as primer pair 16 and HDL17/pKW-rev, two overlapping PCR products were amplified independently from pKWIII/P8.1. The second round of amplifications included both these PCR products as well as the flanking primers T7 and HDL17/pKW-rev. The resulting third PCR product, carrying promoter P8.1, was treated with $S a c$ I and BamHI and cloned into the SmaI and BamHI sites of pKWIII. All pKWIII plasmids carrying mutated or wild-type promoters were transformed into E. coli CMK603 or into E. coli CMK603/pTKRI, expressing Alt and ADPribosylating RNA polymerase.

Synthesis of ONPG 6-phosphate. ONPG 6-phosphate, the substrate for staphylococcal 6-phospho- $\beta$-galactosidase serv- 
ing as an internal standard in promoter strength measurements, was synthesized according to Hengstenberg \& Morse (1969).

Preparation of cell extracts and enzyme assays. Cells of $E$. coli CMK603, harbouring pKWIII with the wild-type or mutant promoter to be tested, or if the reaction of ADPribosylated RNA polymerase was to be tested, this plasmid and pTKRI, (Koch et al., 1995) were grown to $\mathrm{OD}_{590}$ of $0 \cdot 8-1 \cdot 2$ in NB medium with Casamino acids $\left(\mathrm{g} \mathrm{l}^{-1}:\right.$ Lab Lemco Broth, 8; NaCl, 4; Casamino acids, 10) supplemented with ampicillin and kanamycin $(50 \mu \mathrm{g} \mathrm{ml})^{-1}$ each). Cells were harvested and resuspended in phosphate buffer $(10 \mathrm{mM}$ $\mathrm{Na}_{2} \mathrm{HPO}_{4}, 4 \cdot 4 \mathrm{mM} \mathrm{KH}_{2} \mathrm{HPO}_{4}, \mathrm{pH} 7 \cdot 0$ ) and disrupted by sonic oscillation (Branson Sonifier B-12, 2 min, $30 \mathrm{~W}, 50 \%$ duty cycle). After removal of the cell debris the activities of $\beta$ lactamase and 6-phospho- $\beta$-galactosidase were determined according to Wilkens \& Rüger (1994) and Hengstenberg \& Morse (1969). In the $\beta$-lactamase assay initial rates of hydrolysis of the $\beta$-lactam substrate ampicillin $\left(250 \mu \mathrm{g} \mathrm{ml}^{-1}\right)$ were monitored on a Philips PU8735 UV/Vis spectrophotometer by their decreasing $\mathrm{OD}_{233}$ in the presence of aliquots of crude cell extracts in phosphate buffer $(10 \mathrm{mM}$ $\mathrm{Na}_{2} \mathrm{HPO}_{4}, 4.4 \mathrm{mM} \mathrm{KH_{2 }} \mathrm{PO}_{4}, \mathrm{pH} 7 \cdot 0$ ). Initial rates of hydrolysis of ONPG 6-phosphate $(0.3 \mathrm{mM})$ were monitored in phosphate buffer with aliquots of crude cell extracts by increasing $\mathrm{OD}_{412}$.

Promoter strength. The promoter strength, measured in promoter units (PU), was determined as the ratio of the activities of $\beta$-lactamase to 6 -phospho- $\beta$-galactosidase. Every promoter was probed in at least three independent experiments, each in the presence and absence of the phageencoded gene product, Alt. The statistical significance of deviations observed between two promoter strengths was calculated according to Student's Test of Gosset (Leaver \& Thomas, 1977). Significance was accepted if the test resulted in a probability of $\geqslant 99.9 \%$.

Alt assay. The activity of Alt as expressed by vector pTKRI was assayed by radioactive labelling of the target proteins in vitro, according to Rohrer et al. (1975). Incubation mixtures contained in a final volume of $0 \cdot 1 \mathrm{ml} ; 30 \mu \mathrm{g}$ crude cell lysate of Alt-expressing E. coli, $35 \mu \mathrm{g}$ externally added RNA polymerase holoenzyme (optional) and $\left.2.1 \times 10^{5} \mathrm{~Bq}^{[32} \mathrm{P}\right] \mathrm{NAD}^{+}$ (specific activity $1.68 \times 10^{14} \mathrm{~Bq} \mathrm{mmol}^{-1}$ ) in transferase buffer $\left[0.05 \mathrm{M}\right.$ Tris/acetate, $\mathrm{pH} 7 \cdot 5,0.01 \mathrm{M} \mathrm{Mg}(\mathrm{OAc})_{2}, 22 \mathrm{mM}$ $\mathrm{NH}_{4} \mathrm{Cl}, 1 \mathrm{mM}$ EDTA-Na, $0 \cdot 01 \mathrm{M}$ 2-mercaptoethanol, $10 \%$ $(\mathrm{v} / \mathrm{v})$ glycerol]. The specific activity of the $\mathrm{NAD}^{+}$as given is crucial for a proper functioning of the test. The reaction mixture was incubated for $30 \mathrm{~min}$ at $15^{\circ} \mathrm{C}$ and the reaction was stopped by precipitation with $10 \%$ (w/v) TCA. To reduce residual radioactive $\mathrm{NAD}^{+}$, the precipitate was centrifuged, the sediment washed with $70 \%(\mathrm{v} / \mathrm{v})$ acetone/water, dried, dissolved in $80 \mu \mathrm{l}$ electrophoresis sample buffer and incubated at $95^{\circ} \mathrm{C}$ for $5 \mathrm{~min}$. Aliquots of $15 \mu \mathrm{l}$ were applied per channel and separated on a $13 \%$ SDS-polyacrylamide gel (Laemmli, 1970). Gels were stained with Coomassie brilliant blue R, dried and autoradiographed on Fuji-RX films, usually with exposure times of $24 \mathrm{~h}$.

\section{RESULTS}

\section{Probing the expression of the Alt gene product}

The relative strengths of all promoters probed in this test were determined with the aid of the vector pKWIII, a derivative of vector pT7-5 (Tabor \& Richardson, 1985; Wilkens \& Rüger, 1996). The presence and

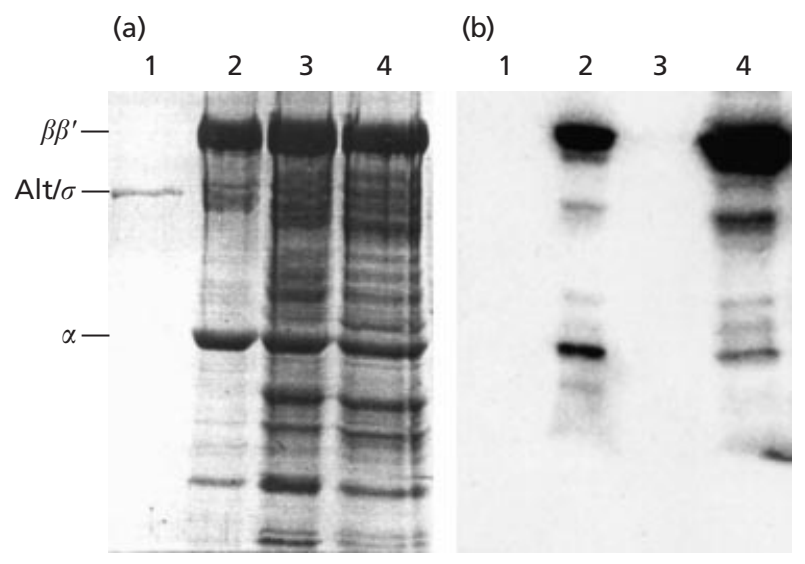

Fig. 2. Constitutive expression of Alt cloned on pTKRI and activity test. (a) A $10 \%$ SDS gel. Lanes: 1, purified Alt; 2, purified RNA polymerase, ADP-ribosylated in the presence of Alt; 3, control - extract of cells grown in the absence of pTKRI, purified and concentrated on a Blue Sepharose column (external RNA polymerase was supplemented for easier identification); 4, extract of cells harbouring pTKRI, purified and concentrated on a Blue Sepharose column (external RNA polymerase was supplemented for easier identification). (b) Autoradiograph of the SDS gel in (a). See Methods for further details.

activity of the promoter-carrying vector pKWIII is detectable as the acquired ampicillin-resistance phenotype of the transformed bacteria and by expression of the enzymes $\beta$-lactamase and 6 -phospho- $\beta$-galactosidase. These enzymes are not a natural part of the E. coli proteome. Therefore, analyses are not disturbed by background activities. The ratios of $\beta$-lactamase, expressed under the control of the different promoters to be tested, to 6-phospho- $\beta$-galactosidase, expressed under the control of an invariant bacterial promoter, reflect the relative promoter strengths (Wilkens \& Rüger, 1994). The co-occurrence of promoter-carrying plasmid pKWIII and vector pTKRI, which expresses Alt constitutively if cloned in E. coli CMK603, constitutes a test system allowing the study in vivo of the transcriptional activities of individual T4 early promoters, in the presence of native or modified RNA polymerase. Transcription from the invariant promoter remains unchanged when probed with modified polymerase.

Since the constitutive expression of Alt, encoded on vector pTKRI, is crucial for the functioning of the test system, the proper synthesis of the enzyme was monitored in different ways. First, the presence of vector pTKRI confers a kanamycin-resistance phenotype upon the transformed host cells. Second, Alt expression may be followed via the growth curves of pTKRI-transformed cells at $\mathrm{OD}_{590}$. The growth of cells expressing Alt lags $1-2 \mathrm{~h}$ behind that of cells carrying the plasmid without an alt insert (data not shown). This growth behaviour may indicate that transcription of E. coli genes by the ADP-ribosylated RNA polymerase is retarded (B. Tiemann \& W. Rüger, unpublished data). Third, ADP-ribosylation catalysed in the presence of Alt can also be followed directly by growing the expressing 
Table 3. Compilation of most of the promoter mutants generated and tested in this study, as well as their activities probed with native and ADP-ribosylated RNA polymerase

The table shows the mutations induced, the individual promoter strengths probed with native and ADP-ribosylated RNA polymerase and the statistical significance of the differences found. 'No' in the significance column means differences without statistical significance; 'yes' means differences in promoter strengths are statistically significant. The designations introduced for the Ptac mutants specify the type of mutation: base pair exchanges in conserved promoter positions of the T4 early promoters are numbered 1-5. The introduction of an A tract is indicated with ' $\mathrm{P}$ ' and the interruption of an A tract by the insertion of $\mathrm{C}$ residues is ' $\mathrm{PC}$ '. The mutants of P8.1 are designated according to the base pair exchange at the corresponding promoter position.

\begin{tabular}{|c|c|c|c|c|c|}
\hline Promoter & -35 & -10 & $\begin{array}{l}\text { Promoter } \\
\text { strength } \\
\text { in the } \\
\text { absence of } \\
\text { Alt (PU) }\end{array}$ & $\begin{array}{l}\text { Promoter } \\
\text { strength } \\
\text { in the } \\
\text { presence of } \\
\text { Alt (PU) }\end{array}$ & Significance \\
\hline Ptac & TCTGAAATGAGCTGTTGACA ATTAATCATCGGCTCG & TATAAT GTGTGG & $16 \cdot 8$ & $17 \cdot 5$ & No \\
\hline M1 & TCTGAAATGAGCTGTT $\underline{\text { TACA ATTAATCATCGGCTCG }}$ & TATAAT GTGTGG & $4 \cdot 25$ & $10 \cdot 6$ & Yes \\
\hline M2 & TCTGAAATGAGCTGTTGACA ATTAATCATCGGGTCG & TATAAT GTGTGG & $10 \cdot 9$ & $17 \cdot 9$ & Yes \\
\hline M3 & TCTGAAATGAGCTGTTGACA ATTAATCATCGGCT $\underline{\mathbf{G} G}$ & TATAAT GTGTGG & 13 & $16 \cdot 4$ & Yes \\
\hline M4 & TCTGAAATGAGCTGTTGACA ATTAATCATCGGCTCG & TACAAT GTGTGG & $11 \cdot 8$ & $12 \cdot 2$ & No \\
\hline M12 & TCTGAAATGAGCTGTTTACA ATTAATCATCGGGTCG & TATAAT GTGTGG & $5 \cdot 35$ & $5 \cdot 45$ & No \\
\hline M13 & TCTGAAATGAGCTGTTTACA ATTAATCATCGGCTGG & TATAAT GTGTGG & $5 \cdot 4$ & $11 \cdot 6$ & Yes \\
\hline M14 & TCTGAAATGAGCTGTTTACA ATTAATCATCGGCTCG & TACAAT GTGTGG & $0 \cdot 9$ & $1 \cdot 9$ & Yes \\
\hline M23 & TCTGAAATGAGCTGTTGACA ATTAATCATCGGGTGG & TATAAT GTGTGG & $17 \cdot 3$ & $17 \cdot 6$ & No \\
\hline M34 & TCTGAAATGAGCTGTTGACA ATTAATCATCGGCTGG & TACAAT GTGTGG & $12 \cdot 6$ & $12 \cdot 5$ & No \\
\hline M123 & TCTGAAATGAGCTGTT $\underline{\mathbf{T} A C A ~ A T T A A T C A T C G G \mathbf{G T}} \overline{\mathbf{G}} \mathrm{G}$ & TATAAT GTGTGG & $7 \cdot 2$ & $10 \cdot 4$ & No \\
\hline M124 & TCTGAAATGAGCTGTT $\underline{-}$ ACA ATTAATCATCGG $\underline{\mathbf{G} T C G}$ & TACAAT GTGTGG & $1 \cdot 2$ & $1 \cdot 4$ & No \\
\hline M134 & 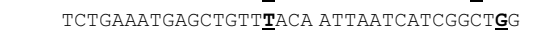 & TACAAT GTGTGG & $7 \cdot 9$ & $8 \cdot 1$ & No \\
\hline M234 & TCTGAAATGAGCTGTTGACA ATTAATCATCGGGTG G & TACAAT GTGTGG & $11 \cdot 4$ & $16 \cdot 3$ & Yes \\
\hline M1234 & TCTGAAATGAGCTGTT $\underline{\mathbf{T} A C A}$ ATTAATCATCGG $\overline{\mathbf{G}} \overline{\mathbf{G}} \mathbf{\mathbf { G }}$ G & TA- $\overline{\mathbf{C}}$ AAT GTGTGG & $5 \cdot 6$ & $6 \cdot 3$ & No \\
\hline M2345 & TCTGAAATGAGCTGTTGACA ATTAATCATCGG $\underline{-} \mathbf{G} \underline{\mathbf{G}}$ & TACTAT GTGTGG & 8 & 8 & No \\
\hline M12345 & TCTGAAATGAGCTGTT & TACTAT GTGTGG & $2 \cdot 9$ & $6 \cdot 3$ & Yes \\
\hline Ptac-P & TCTGAAAAAAGCTGTTGACA ATTAATCATCGGCTCG & TATAAT GTGTGG & $12 \cdot 3$ & $18 \cdot 4$ & Yes \\
\hline M1-P & TCTGAAAAAAGCTGTTTACA ATTAATCATCGGCTCG & TATAAT GTGTGG & 9 & 13.55 & Yes \\
\hline M1-PC & TCTCA & TATAAT GTGTGG & $7 \cdot 5$ & 10 & No \\
\hline M2-P & TCTGAAAAAAGCTGTTGACA ATTAATCATCGGGTCG & TATAAT GTGTGG & $16 \cdot 1$ & $20 \cdot 4$ & Yes \\
\hline M12-P & TCTGAAA $\overline{\text { AAAGCTGTT }}$ ACA ATTAATCATCGG $\overline{\mathbf{G} T C G}$ & TATAAT GTGTGG & $7 \cdot 8$ & $12 \cdot 1$ & No \\
\hline M12-PC & TCTCA $\underline{\mathbf{C}}$ AAAGCTGTTTACA ATTAATCATCGGGTCG & TATAAT GTGTGG & 6 & $8 \cdot 7$ & Yes \\
\hline M14-P & TCTGAAAAAACTGTT & TACAAT GTGTGG & $2 \cdot 3$ & $5 \cdot 1$ & Yes \\
\hline M14-PC & 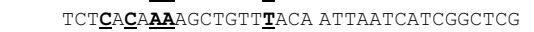 & TA- $\underline{\bar{C}}$ AAT GTGTGG & $8 \cdot 5$ & $8 \cdot 5$ & No \\
\hline M23-P & TCTGAAAAAAGCTGTTGACA ATTAATCATCGGGTGG & TATAAT GTGTGG & $12 \cdot 9$ & $16 \cdot 9$ & Yes \\
\hline M123-P & 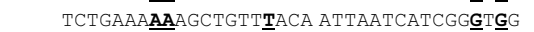 & TATAAT GTGTGG & 8 & $9 \cdot 3$ & No \\
\hline M123-PC & 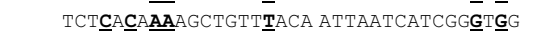 & TATAAT GTGTGG & $7 \cdot 44$ & 13 & Yes \\
\hline M124-P & TCTGAAAAAAGCTGTT TACA ATTAATCATCGG & TACAAT GTGTGG & $2 \cdot 8$ & $5 \cdot 7$ & Yes \\
\hline M1234-P & TCTGAAAAAAGCTGTT & TACAAT GTGTGG & $5 \cdot 1$ & $10 \cdot 9$ & Yes \\
\hline M1234-PC & TCTCACAAAAGCTGTTTACA ATTAATCATCGG & TACAAT GTGTGG & $6 \cdot 6$ & 9 & No \\
\hline P8.1 & CAAAACGATAAAAAGTTGTTTACT TCCTCGGTTAGTTGTGG & TACTAT AACACC & 26 & 52 & Yes \\
\hline P8.1-35a & CAAAACGATAAAAAGTTGT느든 TCCTCGGTTAGTTGTGG & TACTAT AACACC & $5 \cdot 6$ & $10 \cdot 6$ & Yes \\
\hline P8.1-35b & CAAAACGATAAAAAGTTGTTGACT TCCTCGGTTAGTTGTGG & TACTAT AACACC & 19 & $19 \cdot 7$ & No \\
\hline P8.1-42 & CAAAACGATACACAGTTGTTTACT TCCTCGGTTAGTTGTGG & TACTAT AACACC & $7 \cdot 8$ & $7 \cdot 3$ & No \\
\hline P8.1-52 & CACCACGATAAAAAAGTTGTTTACT TCCTCGGTTAGTTGTGG & TACTAT AACACC & $20 \cdot 5$ & 28 & Yes \\
\hline P8.1-42-52 & CACCACGATACACAGTTGTTTACT TCCTCGGTTAGTTGTGG & TACTAT AACACC & $21 \cdot 3$ & $20 \cdot 8$ & No \\
\hline P8.1-4box1 & CAAAACGATAAAAAGTTGTTTACT TCCTCGGTTAGTTGTGG & TACTAT ACGACG & $9 \cdot 6$ & 12 & Yes \\
\hline P8.1-4box2 & CAAAACGATAAAAAGTTGTTTACT TCCTCGGTTAGTTGTGG & TACTAT CCGGGG & $10 \cdot 2$ & $14 \cdot 2$ & Yes \\
\hline
\end{tabular}

cells to a density of $10^{8}$ per ml, lysing the cells (e.g. by sonication) and performing an ADP-ribosylation reaction with the crude cell extract (see Methods). Upon PAGE and autoradiography, a pattern of ADP-ribosylated protein bands was observed. Characteristically, all RNA polymerase bands (and those of other proteins) appear radioactively labelled (Fig. 2). This test may be further enhanced by concentrating the Alt protein from the crude extract on a small chromatography column, containing 1-2 ml Blue Sepharose. Following a washing step, the eluted protein fraction may be tested for its ability to transfer ADP-ribosyl groups to host-cell polypeptides. To facilitate the identification of the radioactively labelled RNA polymerase bands, the reaction mixture was supplemented with commercially available RNA polymerase. Experiments performed with this two-vector system revealed that ADP-ribosylation enhances the mean strengths of most T4 promoters by a factor of up to two (Koch et al., 1995).

\section{Measuring promoter strengths}

Two promoters were selected for this study: promoter Ptac, a chimeric bacterial promoter with fully conserved -10 and -35 regions; and the T4 early promoter, P8.1. Both promoters were chosen because of their strengths within the bacterial and the phage systems, respectively. Though Ptac acts as a very strong bacterial promoter, it is sufficiently different in strength from the viral P8.1 promoter (which is among the strongest T4 promoters), 


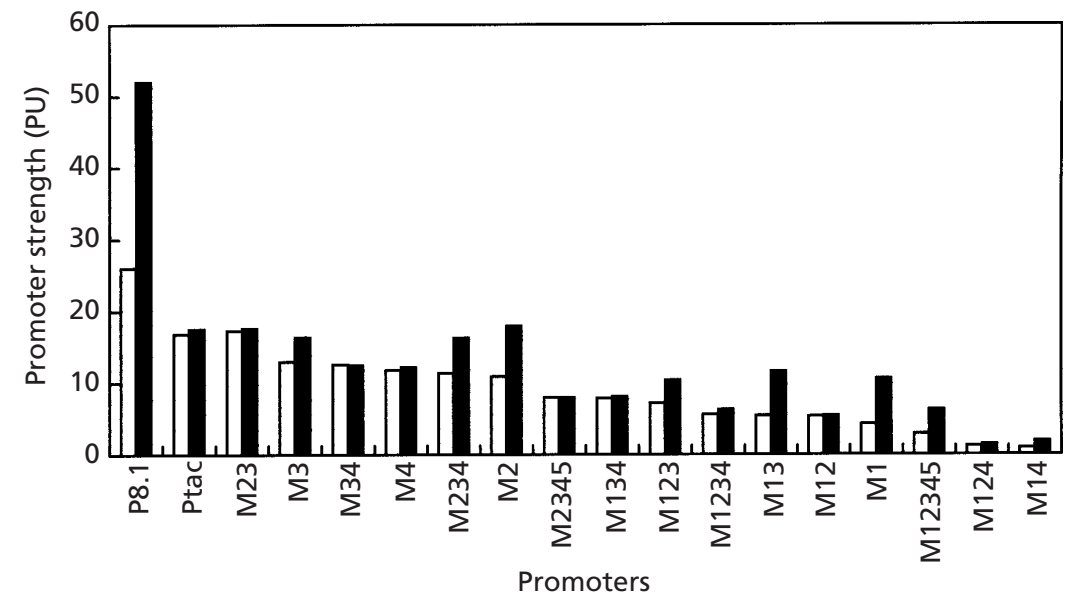

Fig. 3. Activity of promoter P8.1 and Ptac promoter mutants with base pair exchanges in positions highly conserved in T4 early promoters, probed with native $(\square)$ and with Alt-modified (ADP-ribosylated) ( $\square$ ) RNA polymerase.

that differences possibly induced by individual mutations may be easily discerned. The strengths in vivo of all promoters were determined in the presence of native RNA polymerase or its ADP-ribosylated counterpart. Promoter strengths were routinely determined in three to six independent cultures. The resulting values are mean values of all measurements, with maximal deviations of 1 PU. Differences in the individual promoter strengths were tested for their statistical significance, as described in Methods. The results of these measurements for all promoters are compiled in Table 3. To facilitate the interpretation of the individual mutations, the promoter mutants have been classified into three groups. In the first group of mutated Ptac promoters, we placed those with mutations that introduce the highly conserved positions of T4 early promoters. In a second group are Ptac promoter regions with an $\mathrm{A}_{6}$ tract introduced by mutation and we compared the strengths of these promoters to mutants with an interrupted $A_{6}$ tract or with no $A_{6}$ tract around position -42 . Mutations introduced into the P8.1 promoter of phage $\mathrm{T} 4$ are listed in the third group.

\section{Highly conserved positions of early T4 promoters introduced into Ptac}

The test system clearly shows that T4 promoter, P8.1, acts considerably more strongly than the bacterial promoter, Ptac (Fig. 3). In contrast to the reaction of P8.1, the strength of Ptac does not increase when probed with ADP-ribosylated RNA polymerase. The G residues at positions -14 and -16 of Ptac (mutant M23), two positions that are highly conserved among many of the T4 early promoters, also seem to be of importance to the strength of the Ptac promoter, since these two point mutations are the only mutational permutation (M23) in which promoter strength is slightly increased (16.8 vs $17 \cdot 3 \mathrm{PU}$ ). It should be pointed out that when either one of these $G$ residues is altered singly (M2 and M3), a down-regulation of promoter strength results. On the other hand, both single mutants enhance transcription when it is catalysed by ADP-ribosylated RNA polymerase.
Another mutation of interest is the replacement of a $G$ by a $T$ residue at position -32 of Ptac. This mutation changes the E. coli -35 region to the T4 early promoter consensus sequence. However, it decreases the transcription initiation rate of Ptac (M1) considerably. On the other hand, this mutation favours ADP-ribosylated RNA polymerase. The promoter strength of this Ptac mutant is more than doubled if probed with the modified enzyme ( 4.25 vs $10.6 \mathrm{PU})$. All other point mutations introduced into Ptac with the aim of extending the similarity of its consensus sequence to that of the T4 early promoters, result in lower transcription frequencies than those observed with the wild-type promoter. Generally speaking, the single mutations studied here act additively, e.g. mutant M234 is weaker than M23, and M1234 is weaker than M234. However, the introduction of these changes renders most of the Ptac mutants more responsive to ADP-ribosylated RNA polymerase, for example in the promoter mutants M2, M3, M234, M1, M13, M14 and M12345.

\section{Effects of an $A_{6}$ tract introduced into the -42 region of Ptac}

In contrast to the results described above, the introduction of an $\mathrm{A}_{6}$ tract into the sequences of Ptac promoter mutants M1, M2, M12, M14 and M124 leads to a significant increase in their transcriptional activity when probed with native RNA polymerase (Fig. 4). When tested with the altered RNA polymerase, most Ptac promoter mutants carrying a new $A_{6}$ tract at position -42 responded with even further increases in promoter activity (Ptac-P, M124-P, M1234-P, M23-P) or retained their capacity to interact positively with the ADP-ribosylated enzyme (M1-P, M14-P, M2-P). Essentially, promoter strength never decreased after the introduction of the $A_{6}$ tract when probed with ADPribosylated RNA polymerase. However, the interruption of the $\mathrm{A}_{6}$ tract by the introduction of $\mathrm{C}$ residues at positions -43 and -45 was often followed by reduced promoter activity (M1, M12) and loss of sensitivity towards the ADP-ribosylated RNA polymerase (M1, M14, M1234). This effect, however, did not apply to all 


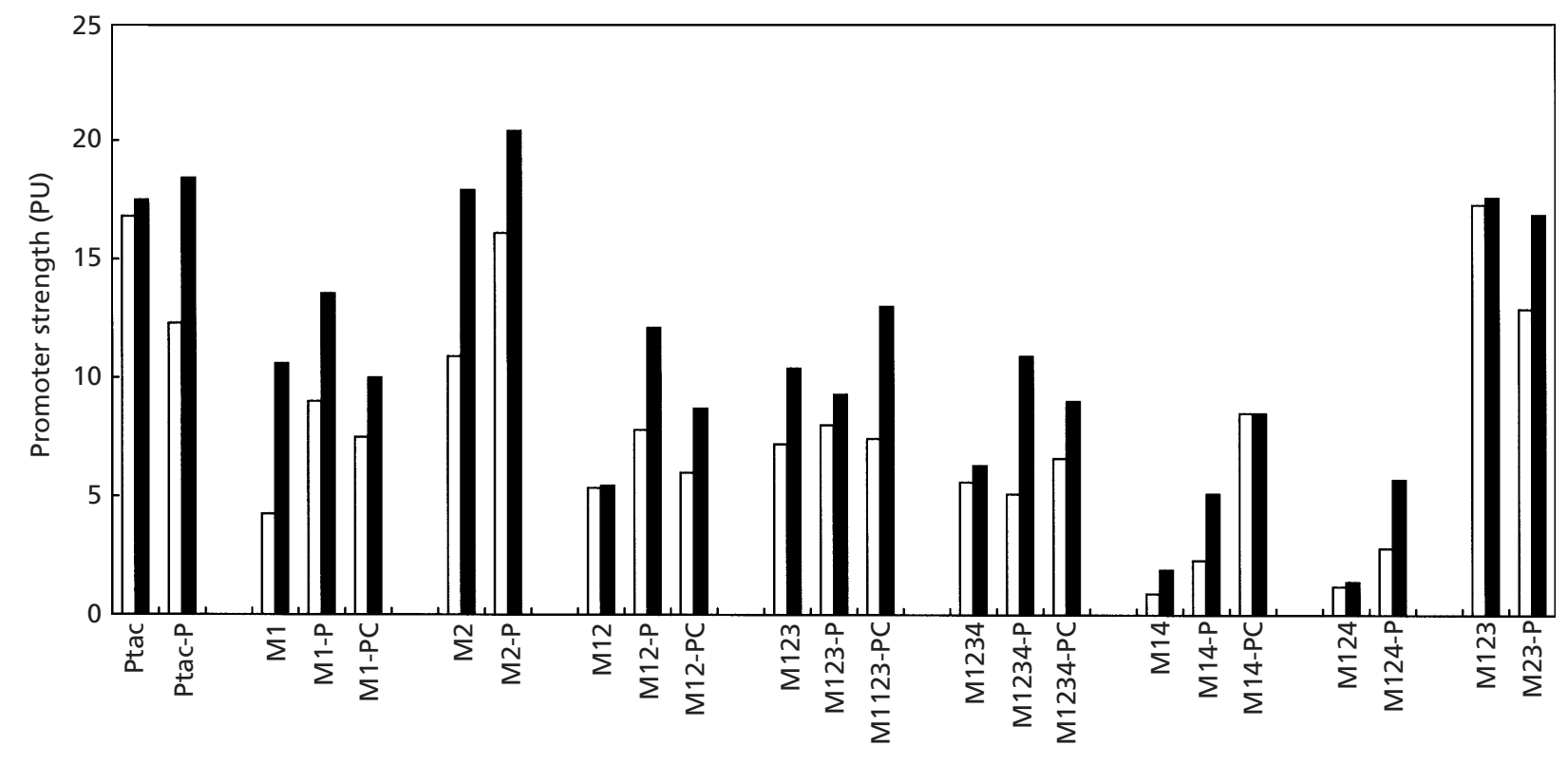

Promoters

Fig. 4. Activity of Ptac promoter mutants without an $A$ tract, with an $A$ tract and with an interrupted $A$ tract, probed with native ( $\square$ ) and with Alt-modified (ADP-ribosylated) ( $\square$ ) RNA polymerase.

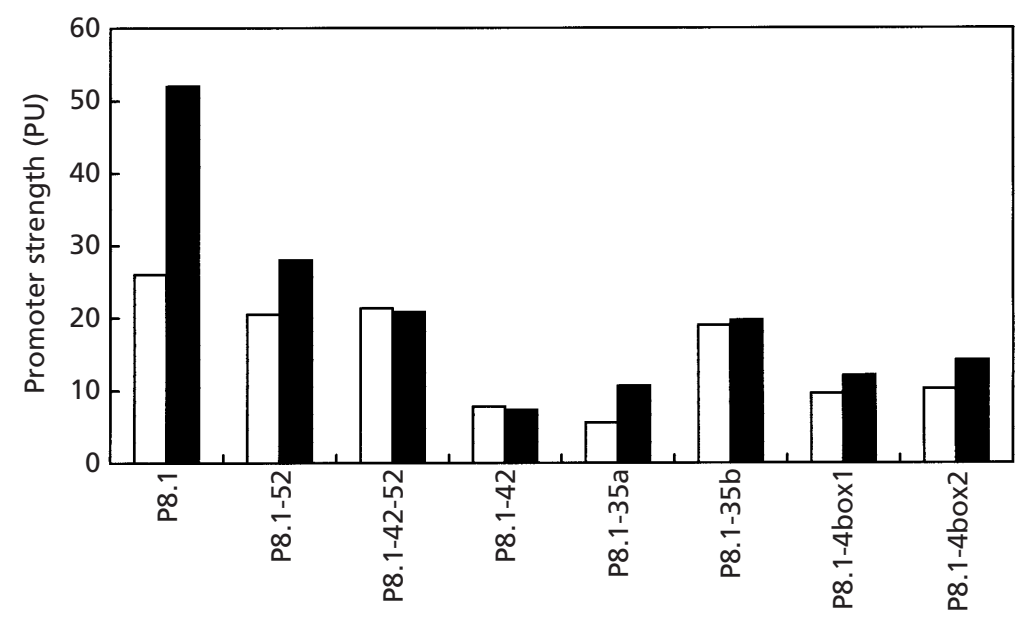

Promoters
Fig. 5. Activity of promoter $P 8.1$ and $P 8.1$ mutants probed with native $(\square)$ and with Alt-modified (ADP-ribosylated) (ם) RNA polymerase. corresponding mutants, although in most cases the transcriptional activity of the native enzyme and of its ADP-ribosylated counterpart increased.

\section{Mutations introduced into the T4 early promoter P8.1}

All base changes introduced into the promoter P8.1 sequence resulted in a decrease in transcriptional activity (Fig. 5). This observation seems logical, since P8.1 is among the strongest T4 promoters. Nevertheless, some of these mutations highlight the importance of certain regions for promoter recognition. Native RNA polymerase recognized promoters P8.1-52 and P8.1-42-52 with a relative high frequency $(20 \cdot 5-21 \cdot 3 \mathrm{PU})$, while interruption of the $A_{5}$ tract in the -42 region (P8.1-42) resulted in low promoter activity $(7 \cdot 8 \mathrm{PU})$. Of these mutants, only P8.1-52 retained the ability to respond to ADP-ribosylated RNA polymerase. However, transcriptional activity was considerably reduced compared to the activity of the P8.1 wild-type promoter, possibly indicating that both $\mathrm{A}$ tracts are necessary to maintain full promoter strength under these conditions. The A tracts in the -52 and -42 regions seem to exert different functions in transcription initiation. The presence of both A tracts in the upstream region seems to be more important for promoter strength when probed with the native RNA polymerase, while the -42 region is more closely associated with changes that allow 
recognition by the modified enzyme. This view is supported by the response of mutants Ptac-P, M2-P and M23-P, all of which show an increase in transcription if probed with modified RNA polymerase.

The mutated promoter P8.1-35a, which is changed at two positions in the T4-specific -35 region $(-32$ and -34), lost transcriptional activity. Nevertheless, it responded with an increase in promoter strength of almost $100 \%$ (from 5.6 to $10.6 \mathrm{PU}$ ) when probed in the presence of ADP-ribosylated RNA polymerase. In contrast, a base change at position -33 (P8.1-35b) responds only moderately when probed with either the native enzyme (19 PU) or with the modified RNA polymerase (19.7 PU). Hence, the T residue at position -33 must be of special importance to the recognition pattern of modified RNA polymerase. Mutations introduced downstream from the -10 region (P8.1-4box1, P8.14 box2), a region that shows conservation among the $\mathrm{T} 4$ early promoters, also resulted in decreased promoter activity. However, these mutants still responded positively to the altered polymerase.

\section{DISCUSSION}

Despite the differences observed between the consensus sequences of bacterial $\sigma^{70}$ and T4 early promoters, both promoters are bound by the identical enzyme, host RNA polymerase. The relative strengths of the T4 early promoters, which compete with a large number of host promoters for the same enzyme molecules in the cellular pool, are reflected theoretically by the very high information content of their consensus sequence and experimentally by the fact that T4 early promoters cannot be cloned without vectors constructed to moderate their activity (Liebig \& Rüger, 1989). Though the apparent strengths of the T4 early promoters are primarily based upon their nucleotide sequences, transcription from these promoters is further increased by ADP-ribosylation of host RNA polymerase, a regulatory step imposed on this enzyme (and on some other host proteins) immediately after infection. The mutation analysis presented here has been conducted with the aim of clarifying those sequence positions that contribute to the differing performance of various promoters and to the different frequencies of transcript initiation observed among bacterial and phage promoters.

\section{Probing promoters on a negatively supercoiled plasmid}

DNA of infecting T4 phage is a $168.9 \mathrm{kbp}$ linear molecule that possibly never reaches a state of substantial underwinding in the course of the replication cycle. This raises the question of whether a T4 early promoter occurring on a $5.3 \mathrm{kbp}$ negatively supercoiled plasmid is an adequate construct with which to probe promoter strengths. Since there are virtually no other means by which to test these promoters in vivo under defined and reproducible conditions, we must accept these limitations. To measure absolute promoter strengths, at least for the T4 early promoter and its mutant derivatives, 5-hydroxymethylcytosine-containing and fully glycosylated DNA would be the only adequate substrate. However, it has been shown that T4 early promoters can be selected on plasmids of similar size on the basis of their strengths, e.g. via the differential expression of $\beta$-galactosidase. Moreover, the relative values obtained previously with the pKWIII/pTKRI system (Wilkens \& Rüger, 1996) and in this study, demonstrate that the system adequately reflects relative promoter strengths. Furthermore, the fact that transcription from T4 promoters increases approximately fourfold compared to transcription from E. coli promoters, must be accepted under the above constraints. A factor of approximately four may not, at first glance, appear exceptional, but if we consider reasonably that not all of the $650 \mathrm{E}$. coli promoters are active at the same time and that transcription of the host genes is rapidly inhibited after infection, this sudden burst of viral transcripts allows T4 phage the opportunity to compete approximately within the same order of magnitude for translation of its transcripts.

\section{Mutations in Ptac}

It has been shown that base pair changes in the -10 and -35 regions of $E$. coli promoters that increase their homology with the consensus sequence, also increase promoter activity (Szoke et al., 1987; Youderian et al., 1982; von Hippel et al., 1984, McClure, 1985). With these examples in mind, a gradual increase in promoter strength can be anticipated with the stepwise transformation of the Ptac sequence to that of the strong T4 early promoter, P8.1. Contrary to this expectation, nearly all base pair exchanges imposed on the Ptac sequence resulted in down-regulation of the promoter, indicating that the intrinsic three-dimensional structures of both promoter elements are quite disparate and do not allow a gradual transition from a weaker E. coli to a stronger T4 phage early promoter by the exchange of single base pairs. Since most base changes also change the twist and slide angles between neighbouring base pairs, mutations also alter the original fit of the threedimensional structures. From this point of view, single base changes or changes involving a few base pairs, which keep angular distortions low, might be more informative than mutants with multiple changes. Nevertheless, a number of the promoter mutants responded conclusively with respect to promoter function and strength.

Promoter P8.1 carries a C residue at position -10 and a $\mathrm{T}$ residue at position -9 . Accordingly, we introduced these nucleotides into the sequence of the Ptac promoter (mutations M4 and M5). However, these changes did not increase the strengths of the bacterial promoters, though both positions are conserved in about $50 \%$ of all T4 early promoters. Since C residues are found more frequently in T4 promoter regions than in E. coli promoters and since these positions are glycosylated in T4 DNA, they may also act as DNA 'identifiers', with a 
possible role in transcription regulation. This hypothesis remains to be tested, as soon as 5-hydroxymethylcytosine phosphoramidites are available to us, to reconstitute T4 early promoters in their original, glycosylated form (Vrielink et al., 1994; Moréra et al., 1999).

A base pair change at position -32 of Ptac, as shown in promoter mutant $\mathrm{M} 1$ and its derivatives, produces a $\mathrm{T}_{3}$ tract found in all -35 regions of $\mathrm{T} 4$ early promoters. This mutation decreased the strength of the Ptac promoter and also suppressed the more positive effects of other mutations, like mutant M123. Mutation experiments conducted by others, to probe this region, revealed a considerable decrease in promoter strength in synthetic promoters after the introduction of $T$ tracts upstream or downstream of the -35 hexamer, as well as within the -35 region (Gaal et al., 1989; BertrandBurggraf et al., 1990; Gartenberg \& Crothers, 1991; Lozinski et al., 1991). The data presented here confirm these earlier findings as far as native RNA polymerase is concerned. However, when probed with ADP-ribosylated RNA polymerase, transcriptional activity increased in all mutants tested, in several cases by more than $100 \%$. This result makes the $\mathrm{T}_{3}$ tract of promoter P8.1 another candidate region for the interaction between the promoter and ADP-ribosylated RNA polymerase.

The phage product Alt transfers the ADP-ribosyl moiety to $\operatorname{Arg}^{265}$ at the carboxy terminus of the $\alpha$-subunit (Ovchinnikov et al., 1977), which is known to interact with promoter upstream elements (Busby \& Ebright, 1994; Estrem et al., 1999). The transfer eliminates the positive charge on the arginine residue and introduces two strongly negatively charged phosphate groups, instead. $\operatorname{Arg}^{265}$ occurs at the amino-terminal part of $\alpha$ helix H1 (Igarashi et al., 1991; Wilkens et al., 1997) and may interact with the DNA helix by forming hydrogen bonds with $G$ residues. The $G$ residues that occur at position -35 of E. coli promoters, and at -36 in T4 promoters, may be possible candidates for such an interaction. Alternatively, the newly introduced charged phosphate groups may also preclude this interaction, by repelling helix $\mathrm{H} 1$ relative to the negatively charged sugar phosphate backbone. As a consequence, the modification of the $\alpha$-subunit may lead to a different recognition of the upstream elements, an idea that is supported by the data presented here. The promoter strength of most Ptac mutants increased after the introduction of an $A_{6}$ tract around position -42 . With two exceptions (M12 and M123), all Ptac promoter mutants carrying an $\mathrm{A}_{6}$ tract showed an increase in promoter strength when probed with ADP-ribosylated RNA polymerase. Conversely, the interruption of the polyA sequence by an intervening $\mathrm{C}$ residue was followed, in most cases, by a loss of this increase.

The length of the spacer in Ptac, comprising only $16 \mathrm{bp}$, is shorter than the typical $17 \mathrm{bp}$ spacer, which also occurs in T4 early promoters. We constructed promoter mutants by inserting an additional nucleotide into the spacer region of Ptac and several of its derivatives (data not shown). In all these cases, homonucleotide tracts were generated, as is frequently observed in T4 early promoters. In most cases, no significant difference in promoter strength was observed and no systematic principle could be deduced. Although Jensen \& Hammer (1998) found an unexpectedly wide range of promoter strengths resulting from variation of the spacer sequence and length, our system did not respond significantly to a variation in the spacer length. Since transcription is attenuated in this test system, it might not be sufficiently sensitive to detect minor changes in promoter strengths.

\section{Mutating P8.1}

To further test the conclusions drawn from the mutation experiments with Ptac, we also mutated several regions in the T4 promoter P8.1. A number of the T4 early promoters show regions conserved downstream from the -10 region, even beyond position +1 . These regions are AT-rich and include some $\mathrm{C}$ residues. In promoter P8.1, this sequence is AACACC. We mutated this region, replacing $\mathrm{C}$ with $\mathrm{G}$ residues (pyrimidines to guanines) or by changing the region to CCGGGG. Both mutations led to promoter down-regulation (P8.1-4box1 and P8.14box 2 ). However, when probed with modified RNA polymerase, the transcriptional activity of the mutated promoter still remained higher than that observed with the native enzyme. The reduction in promoter strengths, in both cases, may be the consequence of the higher GC content of this region, which may interfere with the melting of DNA during the initiation of transcription. Since T4 DNA is AT-rich (64\%), CG base pairs might be required at places to stabilize the DNA duplex.

Another region of importance to T4 early promoter function is the fully conserved $T_{3}$ tract within the -35 region. The importance of this region is substantiated by the decreased strength of mutants P8.1-35. Mutants P8.1-35a and P8.1-35b seem to indicate that the increment in promoter strength as observed in the presence of the modified polymerase is more likely to depend on the $\mathrm{T}$ residue at position -33 , than on the presence of the entire $T_{3}$ tract, as suspected for the corresponding Ptac mutants. However, the $\mathrm{T}_{3}$ tract may also confer a defined structural component upon the -35 region, possibly facilitating neighbouring DNA contacts. Replacement of the central T residue (position -33) would disturb this structure and bring, for instance, the $G$ residue at position -36 and possibly also the -42 region out of alignment, to interact with the ADPribosyl residue or other modified polymerase subunits, respectively (Koch et al., 1995).

Sequence analysis and promoter strength measurements of T4 early promoters revealed that the strongest T4 early promoters not only have an extended -10 region, but also exhibit AT-rich sequences upstream from the -35 region, representing non-canonical B-form features and possibly intrinsic DNA curvatures (Liebig \& Rüger, 1989). In weaker promoters, these regions are missing or have shifted away from these more conserved regions (Wilkens \& Rüger, 1996). Native RNA polymerase and 
the ADP-ribosylated enzyme react differently to an interruption in either one of the $\mathrm{A}$ tracts around positions -42 and/or -52 . Eliminating the A tract around position -42 by the introduction of two interspersed $\mathrm{C}$ residues considerably reduces promoter activity when probed with both enzymes. Moreover, transcription initiated at this mutated promoter is no longer enhanced when probed with ADP-ribosylated polymerase. Elimination of the A tract around position -52 does not change the transcriptional activity of the native polymerase, as compared to that observed with the original promoter sequence. However, transcription by the ADP-ribosylated polymerase is now enhanced. On the other hand, elimination of both A tracts by the replacement of four A residues with four $\mathrm{C}$ residues, when probed with native polymerase, unexpectedly leads to a promoter activity not much weaker than that observed with native polymerase, when acting on the original P8.1 promoter sequence. These four mutations might compensate for structural constraints imposed by these elements on promoter structure and may make the promoter act like a $\mathrm{T} 4$ promoter with no upstream element. Supporting this view, transcription from this mutant promoter is no longer enhanced by the ADP-ribosylated enzyme. Rearrangement of the threedimensional structure may also have occurred in promoter M123-P, where five base pair changes generate a Ptac promoter with 'perfect' -16 and -42 regions. Nevertheless, this promoter acts as a weak promoter with both enzymes.

\section{Conclusions}

The data obtained from more than 40 mutants, probed with native and modified polymerases, reveal that the T4 early promoter exhibits a bipartite structure; it is optimized to interact competitively with native RNA polymerase. This behaviour depends on the conserved and extended -10 region, the absolutely conserved -35 region and, to a minor extent, A runs centred around positions -42 and -52 . Upon interaction with the ADP-ribosylated RNA polymerase, T4 promoter strength is further increased by a factor of about two. This differential performance again depends on the integrity of the extended -10 region. However, more importantly, the modified RNA polymerase and the T4 early promoter regions interact at the T4-specific -35 and the -42 regions, but not at the -52 region. Transcription from an E. coli Ptac promoter that has been mutated experimentally to contain these sequence elements is enhanced if probed with ADP-ribosylated RNA polymerase.

Our data are consistent with the view that, following ADP-ribosylation of one of the two $\alpha$ subunits, only one subunit binds to the -42 region, presumably the unribosylated carboxy-terminal domain. The high conservation and information content of the T4 promoter sequence ensures fast and precise enzyme binding, but the reduced upstream element contacts accelerate the sequence of events, finally leading to open complex formation and a fast promoter clearance. Changes in DNA conformation as a consequence of the new binding pattern and additional ADP-ribosylations, as observed with $\sigma^{70}$ and the other polymerase subunits (Koch et al., 1995), might contribute and accelerate these events, finally leading to the twofold increase in transcription initiation, as observed with the modified RNA polymerase.

\section{ACKNOWLEDGEMENTS}

This research was supported by DFG grant Ru123/22, by grant no. 0310711 of the BMBWFT to W.R. and R.N. and in part by grant 219/2490-1 from the Lithuanian State Program 'Molecular Background of Biotechnology' to R.N.

\section{REFERENCES}

Aiyar, A. \& Leis, J. (1993). Modification of the megaprimer method for PCR mutagenesis: improved amplification of the final product. Biotechniques 14, 366-368.

Barettino, D., Feigenbutz, M., Varcarcel, R. \& Stunnenberg, G. H. (1994). Improved method for PCR-mediated site-directed mutagenesis. Nucleic Acids Res 22, 541-542.

Bertrand-Burggraf, E., Dunand, J., Fuchs, R. P. \& Lefevre, J. F. (1990). Kinetic studies of the modulation of ada promoter activity by upstream elements. EMBO J 9, 2265-2271.

Black, W. B., Showe, M. K. \& Steven, A. C. (1994). Morphogenesis of the T4 head. In Molecular Biology of Bacteriophage T4, pp. 218-258. Edited by J. D. Karam, J. W. Drake, K. N. Kreuzer, G. Mosig, D. H. Hall, F. A. Eiserling, L. W. Black, E. K. Spicer, E. Kutter, K. Carlson \& E. S. Miller. Washington, DC: American Society for Microbiology.

Blattner, F. R., Plunkett, G. I., Bloch, C. A. \& 14 other authors (1997). The complete genome sequence of Escherichia coli K12. Science 277, 1453-1474.

de Boer, H. A., Comstock, L. J. \& Vasser, M. (1983). The tac promotor: A functional hybrid derived from the trp and lac promotors. Proc Natl Acad Sci U S A 80, 21-25.

Busby, S. \& Ebright, R. H. (1994). Promoter structure, promoter recognition, and transcription activation in procaryotes. Cell 79, 743-746.

Estrem, S. T., Ross, W., Gaal, T., Chen, Z. W., Niu, W., Ebright, R. H. \& Gourse, R. L. (1999). Bacterial promoter architecture: subsite structure of UP elements and interactions with the carboxy-terminal domain of the RNA polymerase alpha subunit. Genes Dev 13, 2134-2147.

Gaal, T., Barkei, J., Dickson, R. R., de Boer, H. A., de Haseth, P. L., Alavi, H. \& Gourse, R. L. (1989). Saturation mutagenesis of an Escherichia coli rRNA promoter and initial characterization of promoter variants. J Bacteriol 171, 4852-4861.

Gartenberg, M. R. \& Crothers, D. M. (1991). Synthetic DNA bending sequences increase the rate of in vitro transcription initiation at the Escherichia coli lac promoter. J Mol Biol 219, 217-230.

Goff, C. G. (1974). Chemical structure of a modification of the Escherichia coli ribonucleic acid polymerase alpha polypeptides induced by bacteriophage T4 infection. J Biol Chem 249, 6181 6190.

Hengstenberg, W. \& Morse, M. L. (1969). An improved method of synthesis of $O$-nitrophenyl- $\beta$-D-galactopyranoside 6-phosphate. Carbohydr Res 10, 463-465. 
von Hippel, P., Bear, D. G., Morgan, W. D. \& McSwiggen, J. A. (1984). Protein-nucleic acid interactions in transcription: a molecular analysis. Annu Rev Biochem 53, 389-446.

Horvitz, H. R. (1974). Bacteriophage T4 mutants deficient in alteration and modification of the Escherichia coli RNA polymerase. J Mol Biol 90, 739-750.

Igarashi, K., Fujita, N. \& Ishihama, A. (1991). Identification of a subunit assembly domain in the alpha subunit of Escherichia coli RNA polymerase. J Mol Biol 218, 1-6.

Jensen, P. R. \& Hammer, K. (1998). The sequence of spacers between the consensus sequences modulates the strength of prokaryotic promoters. Appl Environ Microbiol 64, 82-87.

Koch, T., Raudonikiene, A., Wilkens, K. \& Rüger, W. (1995). Overexpression, purification, and characterization of the ADPribosyltransferase (gpAlt) of bacteriophage T4: ADP-ribosylation of E. coli RNA polymerase modulates T4 'early' transcription. Gene Expr 4, 253-264.

Kunkel, T. A. (1985). Rapid and efficient site-specific mutagenesis without phenotypic selection. Proc Natl Acad Sci U S A 82, 488-492.

Kunkel, T. A., Roberts, J. D. \& Zakour, R. A. (1987). Rapid and efficient site-specific mutagenesis without phenotypic selection. Methods Enzymol 154, 367-382.

Laemmli, U. K. (1970). Cleavage of structural proteins during the assembly of the head of bacteriophage T4. Nature 227, 680-685.

Leaver, R. H. \& Thomas, T. R. (1977). Versuchsauswertung: Darstellung und Auswertung experimenteller Ergebnisse in Naturwissenschaft und Technik. Braunschweig: Vieweg.

Liebig, H. D. \& Rüger, W. (1989). Bacteriophage T4 early promoter regions. Consensus sequences of promoters and ribosome-binding sites. J Mol Biol 208, 517-536.

Lozinski, T., Adrych-Rozek, K., Markiewicz, W. T. \& Wierzchowski, K. (1991). Effect of DNA bending in various regions of a consensus-like Escherichia coli promoter on its strength in vivo and structure of the open complex in vitro. Nucleic Acids Res 19, 2947-2953.

McClure, W. R. (1985). Mechanism and control of transcription initiation in prokaryotes. Annu Rev Biochem 54, 171-204.

Moréra, S., Imberty, A., Aschke-Sonnenborn, U., Rüger, W. \& Freemont, P. S. (1999). T4 phage $\beta$-glucosyltransferase: substrate binding and proposed catalytic mechanism. J Mol Biol 292, 717-730.

Norrander, J., Kempe, T. \& Messing, J. (1983). Construction of improved M13 vectors using oligodeoxynucleotide-directed mutagenesis. Gene 26, 101-106.

Ovchinnikov, Y. A., Lipkin, V. M., Modyanov, N. N., Chertov, O. Y. \& Smirnov, Y. V. (1977). Primary structure of alpha-subunit of DNA-dependent RNA polymerase from Escherichia coli. FEBS Lett 76, 108-111.

Perez-Rueda, E., Gralla, J. D. \& Collado-Vides, J. (1998). Genomic position analyses and the transcription machinery. J Mol Biol 275, 165-170.

Rohrer, H., Zillig, W. \& Mailhammer, R. (1975). ADP-ribosylation of DNA-dependent RNA polymerase of Escherichia coli by an
$\mathrm{NAD}^{+}$: protein ADP-ribosyltransferase from bacteriophage T4. Eur J Biochem 60, 227-238.

Saiki, R. K., Gelfand, D. H., Stoffel, S., Scharf, S. J., Higuchi, R., Horn, G. T., Mullis, K. B. \& Erlich, H. A. (1988). Primer-directed enzymatic amplification of DNA with thermostable DNA polymerase. Science 239, 487-491.

Salgado, H., Santos, A., Garza-Ramos, U., van Helden, J., Diaz, E. \& Collado-Vides, J. (1999). RegulonDB (version 2.0): a database on transcriptional regulation in Escherichia coli. Nucleic Acids Res 27, 59-60.

Sambrook, J., Fritsch, E. F. \& Maniatis, T. (1989). Molecular Cloning: a Laboratory Manual, 2nd edn. Cold Spring Harbor, NY : Cold Spring Harbor Laboratory.

Sanger, F., Nicklen, S. \& Coulson, A. R. (1977). DNA sequencing with chain-terminating inhibitors. Proc Natl Acad Sci U S A 74, 5463-5467.

Schneider, T. D., Stormo, G. D., Gold, L. \& Ehrenfeucht, A. (1986). Information content of binding sites on nucleotide sequences. $J$ Mol Biol 188, 415-431.

Siebenlist, U., Simpson, R. B. \& Gilbert, W. (1980). E. coli RNA polymerase interacts homologously with two different promoters. Cell 20, 269-281.

Szoke, P. A., Allen, T. L. \& deHaseth, P. L. (1987). Promoter recognition by Escherichia coli RNA polymerase: effects of base substitutions in the -10 and -35 regions. Biochemistry 26, 6188-6194.

Tabor, S. \& Richardson, C. C. (1985). A bacteriophage T7 RNA polymerase/promoter system for controlled exclusive expression of specific genes. Proc Natl Acad Sci U S A 82, 1074-1078.

Voskuil, M. I. \& Chambliss, G. H. (1998). The -16 region of Bacillus subtilis and other gram-positive bacterial promoters. Nucleic Acids Res 26, 3584-3590.

Vrielink, A., Rüger, W., Driessen, H. P. \& Freemont, P. S. (1994). Crystal structure of the DNA modifying enzyme beta-glucosyltransferase in the presence and absence of the substrate uridine diphosphoglucose. EMBO J 13, 3413-3422.

Wilkens, K. \& Rüger, W. (1994). Transcription from early promotors. In Molecular Biology of Bacteriophage T4, pp. 132-141. Edited by J. D. Karam, J. W. Drake, K. N. Kreuzer, G. Mosig, D. H. Hall, F. A. Eiserling, L. W. Black, E. K. Spicer, E. Kutter, K. Carlson \& E. S. Miller. Washington, DC: American Society for Microbiology.

Wilkens, K. \& Rüger, W. (1996). Characterization of bacteriophage T4 early promoters in vivo with a new promoter probe vector. Plasmid 35, 108-120.

Wilkens, K., Tiemann, B., Bazan, F. \& Rüger, W. (1997). ADP ribosylation and early transcription regulation by bacteriophage T4. Adv Exp Med Biol 419, 71-82.

Yanisch-Perron, C., Vieira, J. \& Messing, J. (1985). Improved M13 phage cloning vectors and host strains : nucleotide sequences of the M13mp18 and pUC19 vectors. Gene 33, 103-119.

Youderian, P., Bouvier, S. \& Susskind, M. M. (1982). Sequence determinants of promoter activity. Cell 30, 843-853.

Received 30 May 2000; revised 10 July 2000; accepted 10 July 2000 